\title{
Die Rolle von 3D-Modellen im Wertschöpfungsprozess von physischen und virtuellen Konsumgütern
}

\author{
Jakob J. Korbel (D) Rüdiger Zarnekow
}

Eingegangen: 18. Juli 2021 / Angenommen: 17. November 2021 / Online publiziert: 9. Dezember 2021

(C) Der/die Autor(en) 2021

Zusammenfassung Die Rolle von 3D-Modellen hat sich für Unternehmen, die sich auf die Wertschöpfung im Bereich der physischen und virtuellen Konsumgüter konzentrieren, in den vergangenen Jahren erheblich verändert. Für herstellende und Handelsunternehmen stellen virtuelle Produkte heute für die Entwicklung und den Vertrieb von physischen Gütern ein essenzielles Medium dar, wobei vermehrt auf virtuelle Umgebungen basierend auf Augmented und Virtual Reality zurückgegriffen wird. Eine Vielzahl an Spieleentwicklern hingegen generiert einen maßgeblichen Teil ihrer Einnahmen nicht mehr durch den Vertrieb ihrer Software, sondern den Verkauf von virtuellen Gütern in ihren Spieleumgebungen. Ziel der Studie ist es, basierend auf einer Literaturanalyse, die Rolle von 3D-Modellen in den Wertschöpfungsprozessen beider Domänen zu beschreiben sowie die durch die beschriebenen Trends entstandenen Ähnlichkeiten und Unterschiede in den Prozessen zu identifizieren, die Möglichkeiten zur Kooperation und dem Austausch von Wissen bieten. Die Ergebnisse implizieren, dass durch den Einsatz von 3D-Modellen über den gesamten Wertschöpfungsprozess neue 3D-Modell-Arten entstanden sind, die mit ihren jeweiligen Abhängigkeiten in einem Wertschöpfungsprozessmodell festgehalten sind. Zudem zeigen die Ergebnisse, dass nicht-funktionale Attribute von 3DModellen von zunehmender Bedeutung sind, die Nutzer eine tragende Rolle in den Wertschöpfungsprozessen spielen und sich Möglichkeiten zur Kooperation hinsichtlich der Erstellung von Augmented und Virtual Reality Umgebungen, der Produktion voneinander abhängiger Güter und dem Austausch von 3D-Modellen basierend auf virtuellen Assets ergeben.

Jakob J. Korbel $(\bowtie) \cdot$ Rüdiger Zarnekow

Fachgebiet Informations- und Kommunikationsmanagement, Institut für Technologie und

Management, Fakultät für Wirtschaft und Management, Technische Universität Berlin, Straße des 17.

Juni 135, 10623 Berlin, Deutschland

E-Mail: jakob.j.korbel@tu-berlin.de 
Schlüsselwörter Virtuelle Produkte · Virtuelle Güter · Virtuelle Assets ·

Hersteller · Handelsunternehmen · Spieleentwicklung · Wertschöpfung

\title{
The Role of 3D Models in the Value Creation Process for Physical and Virtual Consumer Goods
}

\begin{abstract}
The role of 3D models has changed significantly in recent years for companies focused on physical and virtual consumer goods. For manufacturing and retail firms, the use of virtual products and technologies such as augmented and virtual reality are essential for the development and distribution of physical goods. Many game developers, on the other hand, no longer generate the significant part of their revenues through the distribution of their software, but through the sale of virtual goods in their game environments. Based on a literature analysis, the aim of the study is to describe the role of 3D models in the value creation processes of both domains and to identify similarities and differences in the processes which offer opportunities for cooperation and the exchange of knowledge. The results imply that the use of 3D models across the value creation process has created new 3D model types that are described with their respective dependencies in a value creation process model. Furthermore, the results show that non-functional attributes of $3 \mathrm{D}$ models are of increasing importance, as are the users for the value creation processes. Opportunities for cooperation arise with regard to the creation of augmented and virtual reality environments, the production of interdependent goods and the exchange of 3D models based on virtual assets.
\end{abstract}

Keywords Virtual product · Virtual good · Virtual asset · Manufacturing · Retailing · Game development · Value creation

\section{Einleitung}

Digitale 3D-Modelle sind heute aus vielen Branchen nicht mehr wegzudenken und werden bereits in zahlreichen Bereichen eingesetzt, bspw. in der Architektur, der Medizin oder dem Erhalt von Kulturerbe (Bouchlaghem et al. 2005; Rengier et al. 2010; Scopigno et al. 2011). Während Unternehmen in diesen Disziplinen digitale 3D-Modelle vor allem als Mittel zum Zweck nutzen oder sich mit ihren Produkten an Geschäftskunden richten, nutzen Unternehmen in zwei Domänen die Modelle sowohl zur Entwicklung als auch zur Produktion und dem Vertrieb von Gütern, die sich direkt an den Endkunden richten: Herstellende und Handelsunternehmen sowie Spieleentwickler. Unter herstellenden und Handelsunternehmen werden in dieser Studie diejenigen Unternehmen verstanden, die physische Konsumgüter fabrizieren und vertreiben, während sich der Begriff des Spieleentwicklers auf Produzenten von digitalen Computerspielen und virtuellen Welten bezieht.

Als Produzenten und Verkäufer von virtuellen und physischen Konsumgütern wurden diese beiden Domänen lange Zeit als unabhängig voneinander betrachtet, da sich Kollaborationen meist auf Marketingaktionen beschränkten (bspw., Zhu und Chang 2015). Dabei sehen sich die Branchen heute Trends ausgesetzt, die über 
Marketing-Kampagnen hinausgehende Kooperationen ermöglichen könnten: Spieleentwickler stehen auf Grund der stetig zunehmenden Dominanz des Free-to-PlayModells unter Druck, Anreize zu schaffen und Mechanismen einzurichten, die die Spieler zum Kauf virtueller Güter verleiten, da die virtuellen Umgebungen als solche häufig kostenlos sind (Hamari und Keronen 2017; Marder et al. 2019). Herstellende und Handelsunternehmen haben hingegen Augmented Reality (AR) und Virtual Reality (VR) Technologien als Chance erkannt, um ihre 3D-Modell-basierten Produktentwicklungsprozesse zu erweitern und Kunden im Online-Handel die Möglichkeit zu geben, die Güter virtuell zu erleben und anzupassen (Algharabat et al. 2017; Bonetti et al. 2018; Stark et al. 2010). Während das Schaffen von Anreizen und Mechanismen, um Güter an Kundenbedürfnisse anzupassen und Konsumenten in einem kompetitiven Markt zum Kauf zu bewegen, Kernkompetenzen von herstellende und Handelsunternehmen darstellen, gilt dies umgekehrt bei Spieleentwicklern für die Erstellung von 3D Umgebungen, in denen Kunden auf benutzerfreundliche, zielführende und gleichzeitig unterhaltsame Weise mit explizit dafür konstruierten 3D-Modellen interagieren können.

Ziel dieser Studie ist es daher, basierend auf einer systematischen Literaturanalyse nach Webster und Watson (2002), zu untersuchen, (1) welche Rolle 3D-Modellen im Wertschöpfungsprozess virtueller und physischer Güter, unter Berücksichtigung der aktuellen Entwicklungen, zukommt, (2) wie sich diese Entwicklungen auf die Beschaffenheit der 3D-Modelle auswirken und (3) ob die Prozesse Gemeinsamkeiten und Unterschiede aufweisen, die wiederum die Möglichkeit zur Kollaboration und zum Austausch von Wissen und Methoden bieten.

\section{Methodik}

Die Studie basiert auf der Methodik der systematischen Literaturanalyse nach Webster und Watson (2002). Dabei werden relevante Studien zur Thematik identifiziert, sowie anschließend selektiert und kodiert (Abb. 1 und Anhang, Abb. 3), um die Analyse der Publikationen zu ermöglichen.

\subsection{Suche}

Zur Identifikation geeigneter Begriffe für die Suchanfrage wurden in einem ersten Schritt relevante Studien aus dem Bereich der Wertschöpfung virtueller und physischer Güter nach Termen durchsucht, die in den Arbeiten zur Bezeichnung von 3DModellen verwendet werden. Die Analyse ergab, dass für den Term ,3D-Modell““ in Studien zu dieser Thematik Synonyme in Form von spezifischen Begriffen für das Gut bzw. Produkt verwendet wurden. Studien, die ausschließlich den Term „3DModell“" verwendeten, beschäftigten sich hingegen zumeist ausschließlich mit technischen Charakteristika oder Anforderungen an 3D-Modelle als solche, ohne spezifische Informationen hinsichtlich der Wertschöpfungsprozesse. Daher wurde der Term 3D-Modell nicht für die Suche verwendet, sondern die für den Begriff in Bezug auf physische und virtuelle Güter verwendeten Synonyme. Im Bereich der herstellende und Handelsunternehmen wurde bei der Bezeichnung von 3D-Modellen zumeist 


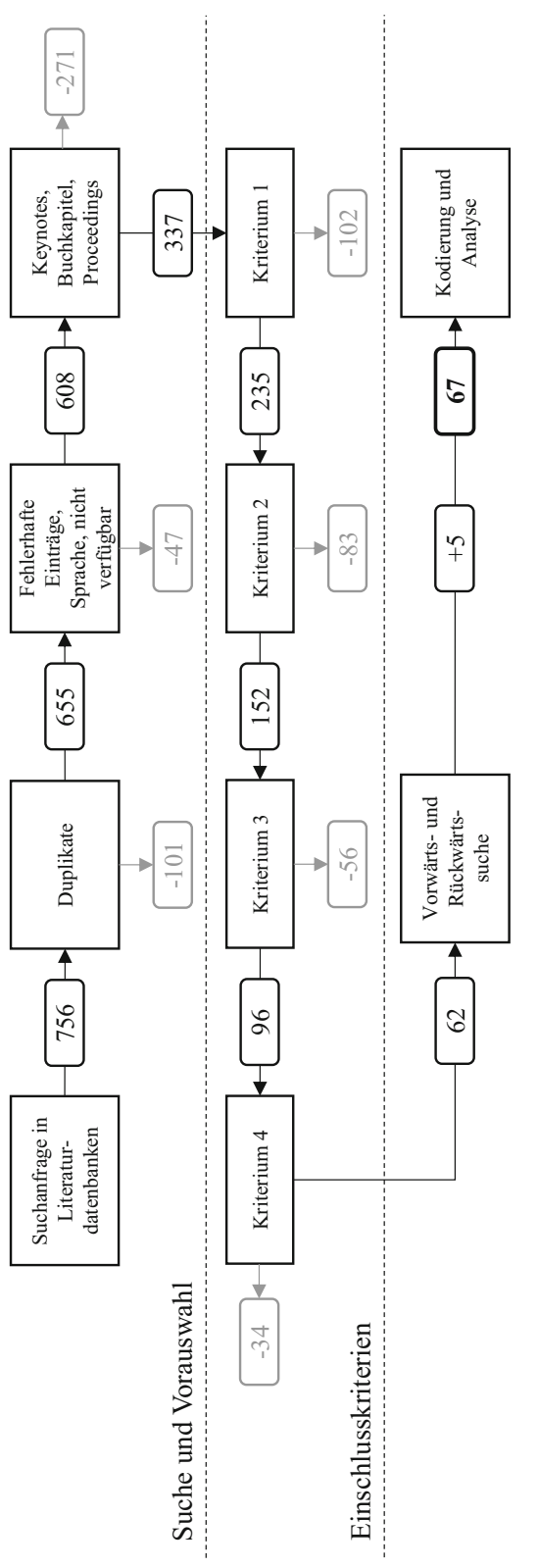

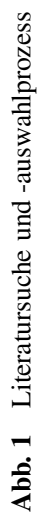


auf den Begriff des virtuellen Produkts (virtual product) verwiesen, im Bereich der Spieleentwickler auf den Begriff des virtuellen Gutes (virtual good). Während beide den Begriff des virtuellen Assets (virtual asset) synonym für diese 3D-Modelle verwendeten, wurde im Fall der Spieleentwickler zusätzlich auf den Begriff ,,virtual item“ und ebenfalls auf den Term ,virtual product“ verwiesen. Da keine weitere Einschränkung in Bezug auf den jeweiligen Schritt innerhalb der Wertschöpfungskette oder den Zeitraum der Publikation vorgenommen werden sollte, resultierte daraus die Suchanfrage ("virtual product*" OR "virtual good*" OR "virtual asset*" OR "virtual item*") im Titel, Abstract und den Keywords der Publikationen. Für den Suchprozess wurden auf Grund ihrer hohen Reputation im Bereich der Wirtschaftsinformatik die Datenbanken WebofScience, ScienceDirect und IEEExplore verwendet. Die erste Anfrage resultierte in 756 Ergebnissen. Anschließend wurden Publikationen entfernt, die als Duplikate vorlagen, in einer anderen Sprache als der Deutschen oder Englischen publiziert und in Büchern, Keynotes und Konferenz-Proceedings veröffentlicht wurden, um eine hohe Qualität der Forschungsergebnisse zu gewährleisten.

\subsection{Einschlusskriterien}

Die verbliebenen 337 Artikel bildeten die Basis für den Selektionsprozess. Hierfür wurden Einschlusskriterien festgesetzt, die zu einem Ausschluss von Artikeln führten, die nicht im Fokus der Studie liegen. Basierend auf den Titeln und Abstracts der Publikationen wurden dabei in einem ersten Schritt Artikel aussortiert, die den Begriff ,virtuell“" nicht in Bezug auf digitale 3D-Modelle, sondern digitale Güter, bspw. Musikdateien, oder virtuelle Hardware, bspw. virtuelle Server, verwendeten. Dieses erste Kriterium führte zum Ausschluss von 102 Publikationen. Das zweite Einschlusskriterium bezieht sich auf das Anwendungsfeld. Auch wenn die Verwendung von 3D-Modellen in anderen Disziplinen, bspw. in der Architektur, interessante Perspektiven auf die Rolle von 3D-Modellen bieten können, konzentriert sich die Verwendung von 3D-Modellen in diesen Bereichen nicht auf die Erstellung und Distribution von Konsumgütern. Die Anwendung dieses Kriteriums führte zum Ausschluss von 83 Publikationen.

In einem letzten Schritt wurde der Volltext der verbleibenden Publikationen gelesen. Dabei wurden Publikationen exkludiert, deren Inhalt keine Rückschlüsse auf die Verwendung der 3D-Modelle als solches, deren Eigenschaften oder die Verwendung innerhalb der Wertschöpfungsprozesse zuließen (Kriterium 3) sowie Studien, die sich auf die Beschreibung des Entwicklungsprozesses von Software-Tools fokussierten (Kriterium 4). Abschließend wurde eine Vorwärts- und Rückwärtssuche basierend auf den Quellen der verbliebenen 62 Studien durchgeführt, um relevante und in dem Prozess nicht berücksichtigte Publikationen zu identifizieren. In diesem Schritt wurden keine Einschränkungen hinsichtlich der Art der Publikation vorgenommen, um auch relevante Publikationen aus Konferenz-Proceedings und Buchkapiteln einschließen zu können. Dabei wurden 5 Studien in die Literaturliste aufgenommen, wodurch sich eine finale Stichprobe von 67 Publikationen ergab, die in den darauffolgenden Schritten kodiert und analysiert wurden. 


\subsection{Literaturkodierung}

Die aus dem Such- und Selektionsprozess hervorgegangenen Studien wurden in Bezug auf die generischen Wertschöpfungsprozesse Entwicklung, Produktion und Vertrieb kodiert und analysiert (Anhang, Abb. 3). Ebenfalls vorab festgelegt wurden die beiden Domänen herstellenden und Handelsunternehmen sowie Spieleentwickler. Im Bereich der herstellenden und Handelsunternehmen konzentrieren sich die meisten Veröffentlichungen auf Konzepte zur Verwendung von 3D-Modellen für die Erstellung von Skizzen, haptische Interaktion sowie das Testen und Bewerten von virtuellen Prototypen. Diese Studien wurden daher unter dem Konzept des Virtual Prototyping zusammengefasst. Ein kleinerer Teil der Literatur befasst sich mit Software-Tools und Plattformen, die eine gemeinsame Arbeit an virtuellen Produkten ermöglichen (Virtual Collaboration). Darüber hinaus beschreiben Publikationen den konkreten Einsatz von 3D-Modellen zur virtuellen Simulation des Produktionsprozesses (Virtual Fabrication) sowie die Einbindung des Nutzers in die Wertschöpfungsprozesse (Virtual Customer Integration) und die Erstellung von Umgebungen, die dem Nutzer ein virtuelles Produkterlebnis ermöglichen (Virtual Product Experience). Im Bereich der Spieleentwickler geben nur wenige Publikationen Auskunft über den Entwicklungsprozess virtuelle Güter (Virtual Prototyping) sowie über die Schritte, die notwendig sind, um ein virtuelles Gut zu produzieren (Virtual Production). Wie im Bereich der herstellenden und Handelsunternehmen beschäftigen sich auch Studien im Bereich der virtuellen Güter mit der Integration der Nutzer in die Wertschöpfungsprozesse (Virtual Customer Integration), wobei eine besondere Form in der autarken Entwicklung und Distribution von Gütern durch den Nutzer besteht (Virtual Entrepreneurship). Der Großteil der Studien beschäftigt sich hingegen mit dem Vertrieb und Konsum von virtuellen Gütern (Virtual Distribution).

\section{Ergebnisse}

Die Ergebnisse orientieren sich an den generischen Wertschöpfungsphasen und sind in Abb. 2 zusammengefasst. Zuallererst wird dabei auf die Produktentwicklung eingegangen, gefolgt von der Produktion der Güter bis hin zu deren Vertrieb.

\subsection{Entwicklung}

In den Entwicklungsprozessen für physische Konsumgüter finden 3D-Modelle im Rahmen des Prototyping breite Anwendung, ebenso wie in der Erstellung von virtuellen Gütern. Physische Konsumgüter stellen dabei jegliche Form von materiellen Gütern dar, die in der realen Welt an Endkunden vertrieben werden, wohingegen virtuelle Güter in ihrer Beschaffenheit immateriell sind, nur in der spezifischen virtuellen Umgebung existieren, für die sie erstellt wurden und auch nur dort einen Nutzen bzw. Wert aufweisen (Fairfield 2005; Hamari und Keronen 2017; Lehdonvirta 2009). Bei virtuellen Gütern handelt es sich somit nicht um 3D-Modelle, die dazu dienen, das Spiel als solches zu produzieren, sondern um Güter, die von Spielern innerhalb der Umgebung genutzt, konsumiert und meist auch gehandelt werden kön- 


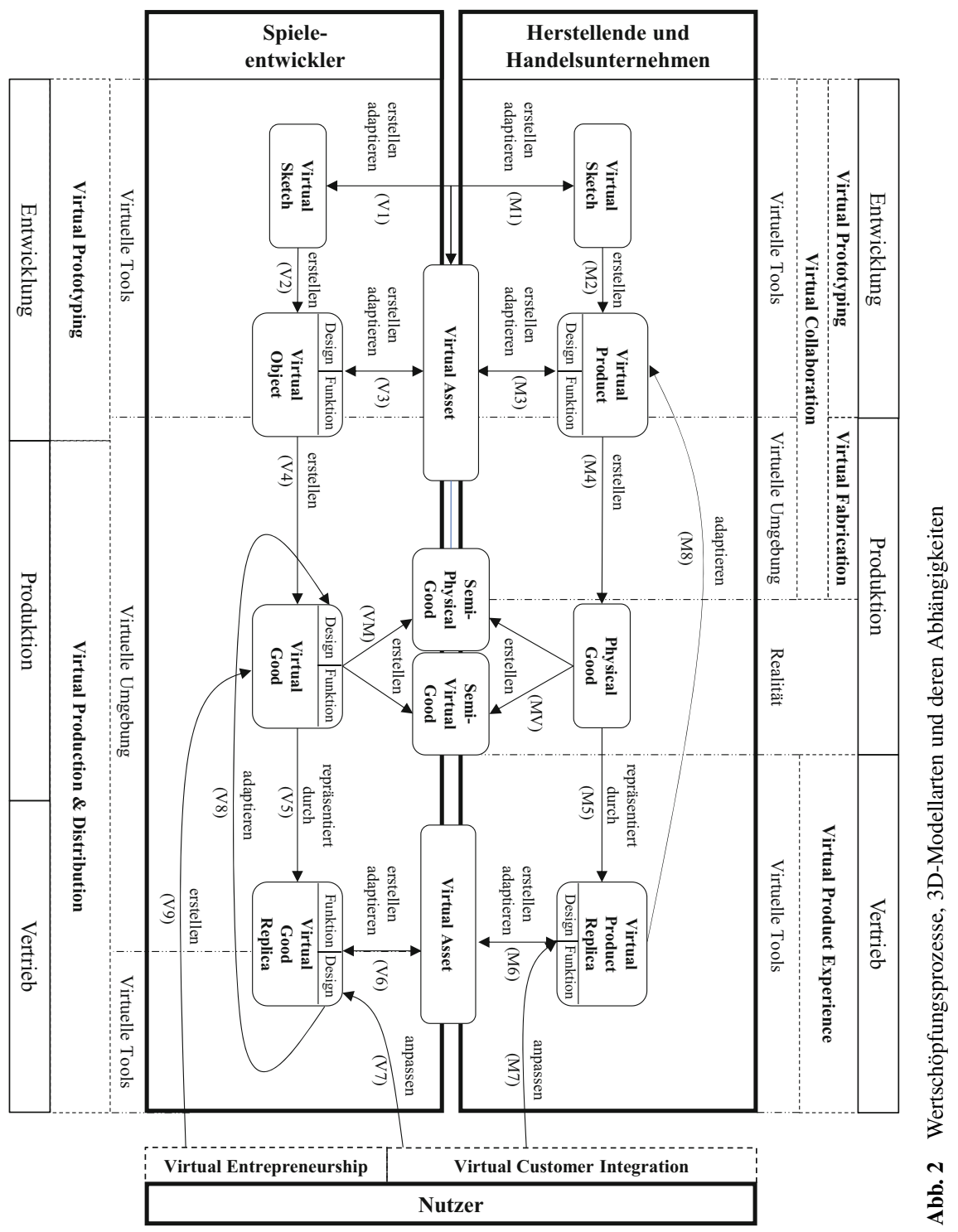


nen. Ebenso handelt es sich bei „virtuellen Produkten“, der gängigen Bezeichnung für 3D-Modelle bei herstellenden und Handelsunternehmen, nicht um 3D-Modelle, die als virtuelles Objekt vertrieben werden, sondern um 3D-Modelle mit Komponenten, Baugruppen und Materialeigenschaften, die unterstützend in den gesamten Wertschöpfungsprozess einbezogen werden, um das zu produzierende physische Gut vorab virtuell gestalten, evaluieren, anpassen und schlussendlich vertreiben zu können (Artacho et al. 2010; Olsen und Saetre 1997; Algharabat et al. 2017; Pfouga und Stjepandić 2018).

Während der Einsatz von 3D-Modellen im Bereich der herstellenden und Handelsunternehmen ausführlich beschrieben ist, konnten im Rahmen dieser Studie nur wenige Publikationen identifiziert werden, die sich mit dem Entwicklungsprozess virtueller Güter auseinandersetzen. Varajão und Morgado (2012) beschreiben den Entwicklungsprozess von virtuellen Gütern aus der Nutzerperspektive, bzw. den Nutzer als Schöpfer des virtuellen Gutes. Dabei wird anhand der virtuellen Welt Second Life exemplarisch dargestellt wie diese entwickelt werden: Die Nutzer erstellen zuallererst eine virtuelle Skizze des virtuellen Gutes. Anschließend können sie die Skizze in ein virtuelles Objekt übertragen, Form, Material und Texturen, also dessen Design festlegen und Skripte schreiben, die die Funktionalität des virtuellen Gutes definieren. Während die Nutzer in diesem Fall in die Lage versetzt werden, die virtuellen Güter nicht nur zu entwickeln, sondern auch eigenständig zu vertreiben, können Spieler im Rahmen von Co-Kreationsansätzen aber auch in die Entwicklung von virtuellen Gütern eingebunden werden, die letzten Endes durch den Spieleentwickler vertrieben werden (Wu und Hsu 2018). Unterstützend kann in dem Entwicklungsprozess dabei bspw. auf die Quality-Function-Deployment Methode zurückgegriffen werden (Li und Kuo 2007), um die Bedürfnisse der Nutzer in Bezug auf das virtuelle Gut zu berücksichtigen.

Ein ähnliches Vorgehen lässt sich im Bereich der herstellenden Unternehmen bei der Entwicklung ihrer Güter feststellen. Auch wenn schlussendlich ein physisches Gut produziert wird, werden im Rahmen des Virtual Prototyping 3D-Modelle, insb. im Zusammenhang mit AR und VR, verwendet, um erste Produktentwürfe zu erstellen, da die Verwendung von virtuellen statt physischen Objekten nicht nur mit geringeren Kosten verbunden ist, sondern auch eine einfache Konfigurierbarkeit und Simulation ermöglicht (Bordegoni et al. 2006). Dabei kann der Einsatz von 3DModellen, im Zusammenhang mit einer VR-Applikation, bereits in der ersten Phase des Entwicklungsprozesses zielführend sein, da die Skizzierung von und Interaktion mit 3D-Objekten die Inspiration fördert und im Vergleich zu 2D-Skizzen zu einer besseren räumlichen Vorstellungskraft führen (Israel et al. 2009). Eben diese Interaktionsmöglichkeit mit 3D-Modellen in VR-Umgebungen führt auch in der darauffolgenden Phase, der Erstellung der virtuellen Prototypen, dazu, dass die Erfolgsrate des Entwicklungsprozesses gesteigert werden kann (Bao et al. 2002; Stark et al. 2010), wobei selbst unerfahrene Nutzer durch die Erweiterung der Umgebung mittels semantischer Schemata in die Lage versetzt werden können, sich schnell in die VR-basierten Entwicklungsprozesse einzuarbeiten (Makris et al. 2012). Ebenso können die 3D-Modelle in den VR-Umgebungen dazu genutzt werden, um die virtuellen Prototypen zu testen und zu evaluieren, wobei die 3D-basierten Prozesse auch hier den klassischen 2D-Verfahren und sogar der Arbeit mit realen Prototypen 
überlegen sind (Park et al. 2008). Studien zeigen aber auch, dass es gewinnbringend sein kann, die 3D-basierten Verfahren mit physischen Prozessen zu verbinden, insb. da sich die menschliche Interaktion mit Produkten nur schwer simulieren lässt. Ein Ansatz ist dabei, die Interaktion mittels haptischer Sensoren zu erfassen und anschließend in die 3D-Modell-Simulation zu integrieren (Bordegoni et al. 2006). Die Haptik führt dabei nicht nur zu einer Erfassung der Nutzerverhaltens, sondern auch zu einem höheren Realitätsempfinden und einer höheren Interaktivität mit dem Prototyp (Teklemariam und Das 2017). Ein anderer Ansatz verkehrt das Prinzip und basiert nicht auf der Übertragung des Physischen in das Virtuelle, sondern des Virtuellen in das Physische. Mittels AR werden dabei die virtuellen Objekte in die reale Umgebung integriert und projiziert, bspw. in die Hand des Nutzers, und dort virtuell verwendet, wodurch sich die Benutzererfahrung, sowie die Leistung im Produktbewertungsprozess verbessern lässt (Park und Moon 2013). Wie im Bereich der virtuellen Güter können auch herstellenden Unternehmen in den Entwicklungsprozessen auf das Wissen ihrer Kunden zurückgreifen (Dahan und Hauser 2002). Neben der Möglichkeit, Nutzerideen und -bewertungen zu Produktdesigns über unternehmensexternen Plattformen wie Online-Communities zu aggregieren (Dahan und Hauser 2002; Bugshan 2015), bieten 3D-Modelle eine kosten- und zeiteffiziente Alternative, um potenziellen Nutzern einen Prototyp des Produkts zu zeigen, dessen Funktionalität und Benutzerfreundlichkeit bewerten zu lassen und Erkenntnisse über die Kaufabsicht der Kunden zu sammeln (Artacho et al. 2010). Der Einsatz von virtuellen Interaktionswerkzeugen erlaubt es den Nutzern dabei, ihre Produktbedürfnisse zu artikulieren und diese Informationen an das Produktentwicklungsteam zu übermitteln (Füller und Matzler 2007; von Hippel und Katz 2002). Da die Immersion mit dem Produkt bei der Produktbeurteilung einen wichtigen Faktor darstellt (Klein 2003), kann auch hier auf VR-Umgebungen zurückgegriffen werden, um die emotionale Bewertung des virtuellen Produktdesigns zu erfassen und verschiedene Designvarianten beurteilen zu lassen (Katicic et al. 2015; Kim et al. 2011).

Die Verwendung von 3D-Modellen im Entwicklungsprozess birgt neben den Potenzialen jedoch auch Herausforderungen, insbesondere in Bezug auf die Komplexität virtueller Produkte. Im Bereich der herstellenden Unternehmen führen unzureichend modellierte Baugruppen und Komponenten sowie deren Abhängigkeiten dazu, dass durch die entstandenen Unsicherheiten, z. B. Funktionalität oder Größe, das Gut nicht nutzbar und somit nicht produzierbar ist (Heimrich und Anderl 2016). Abgemindert werden kann das Auftreten solcher Unsicherheiten durch die Zusammenarbeit von mehreren Entwicklern an ein und demselben virtuellen Produkt; jedoch nur, wenn Software-Tools vorhanden sind, die eine zielführende Kollaboration zulassen. Diese sollten alle Baugruppen als unabhängige Komponenten darstellen, so dass jeder Entwickler auf das entsprechende Objekt zugreifen kann und durch SessionManager-Systeme eine konfliktfreie Bearbeitung der Komponenten und Modelle ermöglicht wird (Rosenman und Wang 2001). Werden diese Systeme zielführend eingesetzt, kann sich dies nicht nur positiv auf die Herausforderungen auswirken, sondern im Gegenzug zu einer Identifikation von Schwachstellen im Produkt führen, die wiederum eine Steigerung der Produkteffizienz ermöglicht (Choi et al. 2002). Damit die entwickelten virtuellen Produkte auch in anderen Wertschöpfungsphasen, 
wie der Produktion, genutzt werden können, sind neben der kollaborativen Entwicklungsumgebung ein für alle Beteiligten nutzbares und einheitliches 3D-Dateiformat und eine IT-Architektur vonnöten, die in der Lage ist, Informationen zu den 3DModellen zwischen allen Beteiligten zu kommunizieren (Zhang et al. 2010; Xiao et al. 2010; Pfouga und Stjepandić 2018, 2015).

\subsection{Produktion}

Die Produktionsprozesse und die Rolle von 3D-Modellen in ebenjenen unterscheiden sich wesentlich in beiden Domänen, da die Produktion von physischen Gütern eine tatsächliche Übersetzung des Gutes in ein reales, materielles Objekt bedingt, während das virtuelle Gut in seiner immateriellen Form verbleibt. Im Bereich der herstellenden Unternehmen nehmen die 3D-Modelle dabei eine unterstützende Funktion ein. Zum einen können auf Basis der eben genannten kollaborativen SoftwareTools und IT-Systeme 3D-Modell-Datenbanken erstellt werden, um wichtige Informationen, wie bspw. die Stückliste oder Bauteilfunktionen, abzurufen und diese Informationen für die Fertigung des Produkts bereitzustellen (Bohm et al. 2005). 3DModelle können aber auch basierend auf additiven Fertigungssystemen die alleinige Grundlage für den Produktionsprozess darstellen, wobei diese Technologien neben Großkonzernen gerade auch Startups und kleineren Unternehmen zugutekommen, die ihre zumeist kleineren Produktionsmengen basierend auf 3D-Druck-Verfahren wirtschaftlich herstellen können (Kang et al. 2018). Zum anderen können 3D-Modelle genutzt werden, um mittels virtueller Fabrikation gesamte Produktionsprozesse vorab zu simulieren und zu testen. Dabei kann mittels AR und VR die Entwicklungs- und Produktionssimulation verknüpft werden, indem die Anwender innerhalb der Umgebung kollaborativ an den für den Produktionsprozess vorgesehenen 3DModellen arbeiten sowie eine Validierung und Verifizierung der 3D-Modelle direkt in der Fabrikumgebung vornehmen können (Dangelmaier et al. 2005).

Wie bereits bei der Entwicklung von virtuellen Gütern, finden sich in der identifizierten Literatur kaum spezifische Angaben zur virtuellen Produktion, also der Transformation des erstellten virtuellen Objekts zu einem in der Spieleumgebung nutzbaren virtuellen Gut. Auch hier lassen sich die Rückschlüsse aus Publikationen zur Erstellung von virtuellen Gütern aus Nutzerperspektive ziehen: Ist das Design des Objekts erstellt, werden diesem in einer Entwicklungsumgebung skript-basiert Funktionalitätseigenschaften zugewiesen (Varajão und Morgado 2012). Der Nutzer kann aber nicht nur eigene virtuelle Güter erstellen (Jung und Pawlowski 2014), sondern ihnen auch Nutzungsrechte zuweisen, die in Berechtigungen wie bspw. Kopieren oder Modifizieren bestehen und es dem Käufer erlauben, Objekte zu vervielfältigen oder sie den eigenen Bedürfnissen anzupassen (Ke et al. 2012). Somit umfasst die Produktion der virtuellen Güter nicht nur das Design und die Funktionalität des Gutes, sondern auch das Setzen von für die Distribution des Gutes relevanten Merkmalen.

Neben dem spezifischen Vorgehen in beiden Bereichen kann die Verzahnung von Physischem und Virtuellem auch zu verschränkten Produktionsprozessen führen. In der Literatur finden sich Beispiele dafür, dass physische oder virtuelle Güter zum Träger des jeweiligen anderen werden können. Virtuelle Güter werden dabei nicht 
nur auf rudimentären Informationsträgern wie CDs vorgehalten, sondern auf physischen Gütern, die im Zusammenhang mit der virtuellen Umgebung stehen. Spieler in der virtuellen Welt Club Penguin konnten bspw. bestimmte virtuelle Spielkarten innerhalb der virtuellen Umgebung nur erhalten, wenn sie die entsprechende physische Sammelkarte kauften (Grimes 2015). Umgekehrten kann auch das virtuelle Gut als Träger für das physische Gut fungieren. Während der Kauf eines virtuellen Gutes, das anschließend mittels additiver Fertigung produziert wird, bereits als virtueller Träger eines physischen Gutes angesehen werden kann, konnte in einem Versuch zu virtuellen Prototypen festgestellt werden, dass Nutzer, denen ein bestimmtes virtuelles T-Shirt angeboten wurde und die dieses kauften, anschließend das entsprechende reale T-Shirt bestellten (Varajão und Morgado 2012). Auch wenn dieses Beispiel das virtuelle Gut nicht als Träger des physischen im eigentlichen Sinne ausweist, deutet es auf das Potenzial eines virtuellen Gutes als Träger eines physischen hin. Unternehmen, die diese Art von Gütern anbieten, müssen somit sowohl auf die materielle als auch die immaterielle Herstellung von Gütern zurückgreifen.

\subsection{Vertrieb}

Die entwickelten und produzierten Güter können anschließend in den Markt eingeführt werden. Dabei zeigen sich auch hier an der materiellen und immateriellen Form der Güter Unterschiede in der Handhabung der Prozesse. Aufgrund ihrer Materialität bedarf es im Markt physischer Güter den stationären und Online-Handel, um die Güter an Endkunden zu vertreiben. Der Online-Handel erlaubt es dabei im Gegensatz zum stationären Handel nicht, mit den Gütern zu interagieren und diese auszuprobieren. Da 2D-Bilder und Textbeschreibungen dem Kunden nicht genügend Informationen über ein Produkt liefern (Lu und Smith 2010), verbinden Kunden den Online-Kauf mit Risiken in Bezug auf die Produktleistung (Yu et al. 2012). 3D-Modelle können hier verwendet werden, um dem Kunden Produktinformationen besser zu kommunizieren (Lu und Smith 2010; Weisstein et al. 2016). Dabei werden 3DModelle des Produkts erstellt, die vom Kunden z. B. in einer AR-Umgebung oder in 360 Grad betrachtet werden können. Das dadurch entstehende virtuelle Produkterlebnis (Yu und Damhorst 2015; Yi et al. 2015) wirkt sich positiv auf das Wissen über das Produkt sowie die wahrgenommene Diagnostik und Flow und damit auf die Zahlungsbereitschaft sowie auf die Kundenzufriedenheit mit der Händler-Website aus (Weisstein et al. 2016; Algharabat et al. 2017; Jiang und Benbasat 2004). Durch die aktuelle Verbreitung der AR-Technologie ist es Nutzern zudem möglich, das Produkt nicht nur auf der Website des Anbieters zu erleben, sondern in der vorgesehenen Umgebung, indem das Produkt in die reale Umgebung projiziert wird (Bonetti et al. 2018), wodurch wiederum die Wahrscheinlichkeit sinkt, eine falsche Kaufentscheidung zu treffen (Lu und Smith 2010); eine Möglichkeit, die auch im stationären Handel nicht gegeben ist. Soll aber eine Einkaufserfahrung im stationären Handel imitiert werden, können 3D-Modelle auch genutzt werden, um basierend auf VR Produkte in einer virtuellen Umgebung zu visualisieren. Die daraus resultierenden Anwendungen führen bei den Nutzern zu einem stärkeren Gefühl von Immersion sowie natürlicher Interaktion beim Online-Shopping (Schnack et al. 2019) und ermöglichen es bspw., unterstützt durch sensorische Daten, passende Kleidungsstücke 
zu finden (Tao et al. 2018). Dabei spielt neben der Visualisierung der Produkte auch die Möglichkeit der Produktanpassung eine Rolle (Olsen und Saetre 1997; Duffy et al. 2004). Bei der Produktanpassung, bspw. der Farbe eines Autos, wird jedoch nicht das virtuelle Produkt innerhalb des Unternehmens geändert, das für die Produktentwicklung verwendet wird, sondern lediglich ein 3D-Objekt zur Verfügung gestellt, welches das Aussehen des Produkts in Übereinstimmung mit Informationen wie Farbe, Größe und Passform verkörpert (Lu und Smith 2010).

Während die Distribution des Gutes bei physischen Konsumgütern nach dem Kauf erfolgt, können virtuelle Güter nicht nur gekauft, sondern innerhalb der Umgebung auch durch Kampf oder das Erfüllen einer bestimmten Aufgabe erlangt werden (Guo und Barnes 2009). Nach dem Erwerb ist das virtuelle Gut rechtlich gesehen jedoch meist nicht im Besitz des Nutzers, sondern noch immer Eigentum der Firma, die die Umgebung betreibt (Strikwerda 2012). Da der Kunde das Gut nicht besitzt, haben die Hersteller der Umgebung das Recht, alle virtuellen Güter eines Spielers zurückzufordern oder Spielerkonten zu schließen (Sheldon 2006). Ausnahmen stellen dabei virtuelle Welten wie Second Life dar, in denen Nutzer, basierend auf dem in der Literatur als Basar-Standard bezeichneten Modell, als virtuelle Entrepreneure agieren können, somit Besitzer ihres Gutes sind und diese auch selbst vertreiben können (Jung und Pawlowski 2015, 2014). Trotz dieser Unterschiede hinsichtlich des Eigentums und obwohl virtuelle Güter zur Kategorie der Informationsgüter gehören (Schmidt 2006), haben virtuelle Güter wesentlich mehr Eigenschaften mit ihrem physischen Pendant als mit herkömmlichen digitalen Gütern gemein, wodurch letztendlich auch der Vertrieb der Güter beeinflusst wird: Wie physische Güter können auch virtuelle Güter eine Knappheit aufweisen (wenn auch künstlich erzeugt), der Besitz mehrerer Kopien desselben virtuellen Gutes können den Nutzen des Konsumenten erhöhen, die Interaktion mit den Gütern führt zu höheren Kaufabsichten und positiven Emotionen gegenüber ebenjenem und die Eigenschaften des Gutes können soziale Distinktionen erzeugen (Fairfield 2005; Lehdonvirta 2009; Ke et al. 2012; Jiang und Benbasat 2004; Zhu und Chang 2015; Animesh et al. 2011; Mäntymäki und Salo 2015; Cleghorn und Griffiths 2015; Cheung et al. 2015). Während die meisten Spieleentwickler Mechanismen eingebaut haben, die es den Nutzern ermöglicht, virtuelle Güter zwischen ihren Accounts zu tauschen, zu handeln und zu übertragen (Jankowski et al. 2016), hat die Wertigkeit von und die Nachfrage nach diesen Gütern dazu geführt, dass Märkte für virtuelle Güter außerhalb der dafür antizipierten Märkte entstanden sind, in denen die Güter direkt von Nutzer zu Nutzer distribuiert werden können. Zum einen werden virtuelle Güter dabei außerhalb der Spieleumgebungen in durch Unternehmen erstellten Märkten gehandelt (bspw. Steam Community Markt), zum anderen sind nutzergetriebene Plattformen entstanden, auf denen die virtuellen Güter mit realem Geld gehandelt werden, die allgemeinhin als Real Money Trading (RMT) Märkte bezeichnet werden (Wang und Chang 2014; Cleghorn und Griffiths 2015; Gumussoy 2016; Guo und Barnes 2009, 2011; Hassouneh und Brengman 2011; Lehdonvirta et al. 2009). Der Handel auf diesen Märkten, der mitunter kritisch zu betrachten ist (Heimo et al. 2018), lässt sich besonders bei so genannten kosmetischen bzw. nicht-funktionalen virtuellen Gütern beobachten, die dem Spieler keinen Wettbewerbsvorteil verschaffen, sondern sich durch ihr Aussehen unterscheiden und wegen der symbolischen Bedeutungen, 
die das Gut vermitteln, gekauft werden (Shang et al. 2012; Hamari und Keronen 2017). Nutzer drücken sich durch diese Güter aus, indem sie bspw. ihre virtuellen Räume dekorieren oder ihre Avatare einkleiden (Mäntymäki und Salo 2015). Diese nicht-funktionalen Attribute haben in den letzten Jahren an Relevanz gewonnen, da kosmetische Güter heute als die Haupteinnahmequelle von Free-to-Play-Spielen angesehen werden können (Marder et al. 2019; Hamari und Keronen 2017).

\section{Diskussion und Implikationen}

Die Ergebnisse der Literaturanalyse zeigen, dass 3D-Modelle im gesamten Wertschöpfungsprozess physischer und virtueller Güter eingesetzt werden können, um virtuelle Güter $\mathrm{zu}$ entwickeln, zu produzieren und $\mathrm{zu}$ distribuieren und die Entwicklung physischer Güter zu erleichtern sowie deren Produktion zu simulieren und Endkunden die Möglichkeit zu geben, die Produkte zu individualisieren und im Rahmen des Vertriebs erlebbar zu machen. Dabei kann die Einbindung des Nutzers als Tester, Gestalter oder gar als Entrepreneur von entscheidender Bedeutung sein. Die Ergebnisse zeigen auch, dass die dabei eingesetzten 3D-Modelle sich in ihrer Funktion, Komplexität und Verwendung in einem Maße unterscheiden, die eine Kategorisierung bedingt und ermöglicht, die über die in der Literatur verwendeten Begriffe des virtuellen Produkts und des virtuellen Gutes hinausgeht (Abb. 2 und Tab. 1).

In der Entwicklung sowohl von virtuellen als auch physischen Gütern wird auf virtuelles Skizzieren zurückgegriffen, um erste Entwürfe von Prototypen zu erstellen, wobei herstellende Unternehmen nicht nur in diesem Entwicklungsschritt, sondern auch in den darauffolgenden AR- und VR-basierte Ansätze verfolgen, die durch die verbesserte räumliche Vorstellungskraft, ein höheres Realitätsempfinden sowie die Interaktivität mit dem Prototyp, zu einer zielführenderen Benutzererfahrung und höheren Erfolgsraten im Entwicklungsprozess führen und damit physischen Prototypen überlegen sind (Bao et al. 2002; Stark et al. 2010; Israel et al. 2009; Park et al. 2008; Park und Moon 2013; Teklemariam und Das 2017). Durch den Einsatz von AR und VR verlagern die herstellenden Unternehmen dabei implizit ihre Produktentwicklung in virtuelle Umgebungen, wobei die Kernkompetenzen von Spieleentwicklern

Tab. 1 Anwendungsbeispiele

\begin{tabular}{lll}
\hline 3D-Modell & Anwendungsbeispiel & Link \\
\hline Virtual Sketch & Gravity Sketch VR & gravitysketch.com \\
Virtual Object & OpenBrush VR & openbrush.app \\
Virtual Product & Autodesk Fusion360 & autodesk.de/products/fusion-360 \\
Virtual Product Replica & IKEA Virtual Interior Designer & present.digital/ikea \\
Virtual Good & Epic Games' Fortnite & epicgames.com/fortnite \\
Virtual Good Replica & Steam Workshop & steamcommunity.com/workshop \\
Virtual Asset & Sketchfab & sketchfab.com \\
Semi-virtual Product & Nintendo's amiibo & nintendo.com/amiibo \\
Semi-physical Product & - & - \\
\hline
\end{tabular}


essenziell sind: Interaktivität, Usability und User Engagement (Algharabat et al. 2017). Daher kann eine Zusammenarbeit mit Spieleentwicklern oder eine Übernahme von Produktentwicklungsmethoden von ebenjenen die Vorteile fördern, die sich aus der Anwendung von AR und VR bei herstellenden Unternehmen ergeben. Die im Rahmen der Entwicklungsprozesse erzeugten 3D-Skizzen und -Modelle werden dabei in digitalen Datenbanken gespeichert, vorgehalten und ausgetauscht (Xiao et al. 2010; Zhang et al. 2010) und sind damit auch in anderen Phasen des Wertschöpfungsprozesses nutzbar. Während also die virtuellen Skizzen die Grundlage für die Erstellung virtueller Produkte bilden (Abb. 2, M2), lassen sich beide als virtuelle Assets betrachten, die in verschiedenen Phasen des Produktentwicklungsprozesses genutzt und angepasst werden können (Abb. 2, M1, M3). Gleiches gilt für die virtuellen Skizzen und Objekte im Bereich der Spieleentwickler, die in nachfolgenden Phasen des virtuellen Entwicklungs- und Produktionsprozesses übernommen werden können (Varajão und Morgado 2012) und als Grundlage für virtuelle Güter dienen, wenn sie in die entsprechende virtuelle Umgebung integriert werden (Abb. 2, V1-V4). Im Entwicklungsprozess von Spieleentwicklern könnte es dabei zielführend sein, Produktentwicklungsmethoden von herstellenden Unternehmen zu übernehmen, um die virtuellen Güter besser an die Kundenbedürfnisse anzupassen, wie bereits von Li und Kuo (2007) untersucht, sowie die Erstellung ihrer virtuellen Güter und Objekte durch den Einsatz von VR effizienter zu gestalten.

Im darauffolgenden Produktionsprozess dienen 3D-Modelle im Bereich der herstellenden Unternehmen als Hilfsmittel, während 3D-Modelle im Bereich der Spieleentwickler das tatsächliche virtuelle Gut und damit den Fokus des Absatzes darstellen. Während Nutzer in virtuellen Welten das Gut nicht nur entwickeln, sondern eigenständig produzieren und schlussendlich vertreiben können (Abb. 2, V9), bieten herstellende und Handelsunternehmen dem Nutzer die Möglichkeit die Eigenschaften des Produkts individuell anzupassen (Abb. 2, M7, M8), nicht aber alle weiteren Wertschöpfungsprozesse selbstständig durchzuführen. Aktuelle Entwicklungen im Bereich der additiven Fertigung (Kang et al. 2018) könnten Nutzer jedoch auch im Bereich der herstellenden Unternehmen mittels 3D-Modellen in die Lage versetzen, eigene physische Güter zu erstellen, wobei dem herstellenden Unternehmen schlicht die Funktion des Fertigungsunternehmens zukäme. Beispiele hierfür sind Unternehmen wie i.materialize, die den Entwicklungs- und Vertriebsprozess in die Hände des Nutzers legen und die auf der Plattform zur Verfügung gestellten Güter der Nutzer bei einer Bestellung für die Endkunden produzieren. Neben dem physischen und virtuellen Gut als solchem können die Güter aber auch als Träger des jeweils anderen genutzt werden und somit zu einer Verschränkung der Produktionsprozesse führen. Die dabei entstehenden Produkte werden in dieser Studie als semi-virtuell bezeichnet, wenn das physische Gut der Träger des virtuellen ist, und im umgekehrten Fall als semi-physisch (Abb. 2, VM, MV). Ein Beispiel für Ersteres sind die von Nintendo erfolgreich vertriebenen amiibo-Figuren (Tab. 1). Für Zweiteres finden sich in der Praxis hingegen kaum Beispiele. Herstellende Unternehmen und Spieleentwickler könnten hier eine Zusammenarbeit in Erwägung ziehen, um den Absatz physischer Güter zu fördern und die Anzahl an Spielern in der virtuellen Umgebung zu erhöhen, indem die Nutzer physische Güter nur durch den Kauf von virtuellen Gütern erwerben können. Die Erstellung dieser Arten von Gütern bedingt dabei die 
Berücksichtigung sowohl der physischen als auch der virtuellen Produktionsprozesse und bietet somit die Möglichkeit zur Kooperation zwischen Spieleentwicklern und herstellenden Unternehmen.

Auch durch die Distributionsprozesse in beiden Bereichen entstehen Arten von 3D-Modellen, die sich von den in der Literatur beschriebenen unterscheiden. In beiden Bereichen sind dabei die nicht-funktionalen Attribute des 3D-Modells von zunehmender Relevanz. Die meisten Entwickler von virtuellen Welten und Spielen generieren heute Einnahmen durch den Verkauf von nicht-funktionalen Gütern, wobei deren Wertigkeit zur Entstehung von Märkten außerhalb der Spieleumgebung beigetragen hat, in denen die Güter zwischen den Nutzern mit realer oder virtueller Währung gehandelt werden (Cleghorn und Griffiths 2015; Wang und Chang 2014). Die hier gehandelten 3D-Modelle stellen in diesem Prozess jedoch keine virtuellen Güter dar, sondern lassen sich ebenfalls als virtuelle Assets bezeichnen, da sie außerhalb der Umgebung in einem Sekundärmarkt gehandelt werden und die Absicht nicht auf der Nutzung und dem Konsum des Gutes innerhalb der Umgebung liegt, sondern in der Generierung von Profit basierend auf dessen Rarität oder Eigenschaften in der virtuellen Umgebung (Abb. 2, V6). Abgesehen von dem nutzergetriebenen Handel mit virtuellen Gütern können 3D-Modelle aber auch verwendet werden, um den Nutzern eine individuelle Anpassung des Gutes zu ermöglichen. Dafür werden Replikate der virtuellen Güter erzeugt, die nicht alle Attribute der virtuellen Güter in der Umgebung enthalten, bspw. deren Funktionalität, und nur die Manipulation gewisser Eigenschaften durch den Nutzer zulassen. Ein Beispiel für diesen Prozess ist die Steam-Workshop-Umgebung (Tab. 1), die es Nutzern von virtuellen Welten und Spielern u.a. ermöglicht, nicht-funktionale Designs, bspw. für Hüte zu erstellen und diese dort zur Verfügung zu stellen. Die daraus hervorgehenden Designs können anschließend vom Spieleentwickler übernommen und auf das virtuelle Gut im Spiel übertragen werden (Abb. 2, V5, V6). Um die Effizienz dieser nutzerzentrierten Prozesse zu steigern, sollten Spieleentwickler den Einsatz von VR-basierten Produktentwicklungstools für ihre Nutzer evaluieren, da Studien im Bereich der herstellenden Unternehmen zeigen, dass diese für die Erstellung und Evaluation von 3D-Modellen durch Nutzer zielführend eingesetzt werden können. Des Weiteren bietet sich in diesem Zusammenhang die Kooperation mit herstellenden Unternehmen hinsichtlich des Austausches von 3D-Objekten an. Herstellende Unternehmen verfügen über ein variantenreiches Repertoire an virtuellen Produkten. Eine Überführung dieser virtuellen Produkte in virtuelle Assets würde Spieleentwickler dazu befähigen, diese für die Entwicklung ihrer Umgebungen oder virtueller Güter zu nutzen. Von dieser Art der Kooperation würden auch herstellende Unternehmen profitieren, da diese durch die Produktplatzierung in den Umgebungen MarketingEffekt erzielen könnten.

Jedoch stellt ebenjene Überführung von virtuellen Produkten in virtuelle Assets aufgrund der Eigenschaften virtueller Produkte eine Herausforderung für herstellende und Handelsunternehmen dar. Da virtuelle Produkte komplexe Komponenten und Materialeigenschaften beinhalten, um bspw. eine Simulation des physischen Produkts zu ermöglichen, sind virtuelle Produkte nicht direkt für die Verwendung in für Vertriebszwecke erstellte virtuelle Umgebungen geeignet. Es müssen Replikate der virtuellen Produkte erstellt werden (Abb. 2, M5), indem die 3D-Modelle 
durch Vernachlässigung oder Vereinfachung von Komponenten heruntergerechnet werden (Lee et al. 2001), um ein Reengineering der Produkte zu vermeiden und sie in virtuellen Umgebungen nutzbar zu machen. Dabei handelt es sich wiederum um eine Kernkompetenz von Spieleentwicklern. Daher bieten sich die Übertragung von Methoden und Kooperationen zur Erstellung der 3D-Modelle und virtuellen Umgebungen aus dem Bereich der Spieleentwickler in den der herstellenden und Handelsunternehmen an. Nach dem durch das virtuelle Produktreplikat unterstützten Kaufprozess erhält der Kunde nicht das 3D-Modell, sondern das tatsächliche physische Produkt. Somit stellt dieses im Gegensatz zu virtuellen Assets selbst kein Handelsobjekt dar. Die virtuellen Produktreplikate können jedoch als virtuelle Assets für herstellende und Handelsunternehmen übernommen und bei Bedarf während des gesamten Produktentwicklungsprozesses verwendet werden, bspw. für Nutzerstudien in der Entwicklungsphase (Abb. 2, M6). Da diese Art von 3D-Modellen für den Einsatz von AR- und VR-Anwendungen unabdinglich ist, sollten herstellende Unternehmen evaluieren, in welchem Schritt ihrer Wertschöpfung die Erstellung von virtuellen Produktreplikaten zielführend ist, um eine nachgelagerte, kosten- und zeitintensive Anfertigung dieser 3D-Modell durch externe Dienstleister zu vermeiden. Für Handelsunternehmen stellt die Erstellung von virtuellen Produktreplikaten eine weitaus größere Herausforderung dar, da die Unternehmen selbst nicht in die Entwicklung des Produkts involviert sind und somit auch über keinerlei 3D-Modelle verfügen. Insbesondere ergibt sich hier die Notwendigkeit von virtuellen Produktreplikaten vor dem Hintergrund der anhaltenden Diffusion der AR-Technologie im Online-Handel. Während sich die Umsetzung von AR-Umgebungen als solches aufgrund des breiten Angebots von Dienstleistern und der ausreichend vorhandenen Anzahl an geeigneten Frameworks heute kosteneffizient bewerkstelligen lässt, sind diese ohne die zu den Produkten passenden 3D-Modelle nicht nutzbar. Daraus ergibt sich die Notwendigkeit von auf virtuelle Produktreplikate spezialisierte Online-Plattformen, die ebenjene Art von 3D-Modellen unter der Verwendung von eindeutigen Identifikationsmechanismen zur Verfügung stellen sowie Geschäftsmöglichkeiten für technische Künstler, Designstudios und Software-Tools, die sich auf die Erstellung von virtuellen Produktreplikate spezialisieren.

\section{Zusammenfassung}

Die Studie hatte zum Ziel, die Rolle von 3D-Modellen im Wertschöpfungsprozess virtueller und physischer Güter unter der Berücksichtigung aktueller Trends zu untersuchen und zu diskutieren, ob in diesen Prozessen Unterschiede und Gemeinsamkeiten existieren, die eine Kollaboration und den Austausch von Wissen ermöglichen. Die Ergebnisse stellen ebenjene Rolle von 3D-Modellen über den gesamten Wertschöpfungsprozesse für beide Arten von Gütern dar und sind abschließend unter Berücksichtigung der jeweiligen Abhängigkeiten in Abb. 2 zusammengefasst. Dabei wird ersichtlich, dass 3D-Modelle insb. im Zusammenhang mit AR und VR für herstellende und Handelsunternehmen das Potenzial bieten, die Entwicklungsprozesse effizienter und kostengünstiger zu gestalten sowie neue Formen der Produktionssimulation zu nutzen und den Vertrieb der Güter an die Kunden zu erleichtern. 
Spieleentwickler hingegen nutzen 3D-Modelle nicht nur im gesamten Wertschöpfungsprozess, sie stellen für viele Entwickler heute in Form von virtuellen Gütern auch die primäre Einnahmequelle dar, wobei die nicht-funktionalen Eigenschaften dieser Güter an Relevanz gewinnen. In beiden Bereichen nimmt dabei der Nutzer eine tragende Rolle ein, da sie/er sich als virtueller Entrepreneur, als Ersteller von 3D-Modell-Attributen oder als Tester und Bewerter von virtuellen und physischen Gütern in die Entwicklungs-, Produktions- und Vertriebsprozesse einbringen kann.

Die Diskussion der Ergebnisse zeigt zudem, dass sich die 3D-Modelle in den verschiedenen Wertschöpfungsphasen in einem Maße unterscheiden, die eine Kategorisierung der 3D-Modelle über die in der Literatur verwendeten Begriffe erlaubt sowie Kooperationen und den Austausch von Wissen und Methoden ermöglicht: Spieleentwickler können von Kooperationen und dem Austausch von Methoden profitieren, da so die Anpassung ihrer virtuellen Güter an die Kundenbedürfnisse verbessert werden kann. Zudem bietet es sich, basierend auf Ergebnissen im Bereich der herstellenden Unternehmen, an, VR-basierte Entwicklungstools für die eigenen Produktentwicklungsphasen zu verwenden sowie den Nutzern ebenjene VR-Tools für die Erstellung von virtuellen Güterreplikaten zur Verfügung zu stellen. Des Weiteren empfiehlt es sich, in Kooperationen mit herstellenden Unternehmen deren in virtuelle Assets überführte virtuelle Produkte als virtuelle Güter in ihre Umgebungen zu integrieren. Von Letzterem würden auch herstellende Unternehmen in Form eines Marketing-Effekts profitieren, da ihre Produkte in den virtuellen Umgebungen platziert würden. Herstellende Unternehmen könnten zudem von einem Wissensaustausch mit Spieleentwicklern profitieren, um ihre AR- und VR-Umgebungen sowohl in der Entwicklung als auch im Vertrieb an die Nutzerbedürfnisse anzupassen. Dabei müssen sie für deren Implementierung notwendigerweise auf virtuelle Produktreplikate zurückgreifen und Methoden entwickeln, die eine einfache Überführung ihrer virtuellen Produkte in virtuelle Produktreplikate innerhalb ihrer Wertschöpfungskette ermöglichen. Ohne virtuelle Produktreplikate kann insb. die im Handel aufkommende Darstellung der Produkte mittels AR-Technologie nicht umgesetzt werden. Ambitionen von Firmen wie Facebook, die die Schaffung eines VR-basierten Metaversums vorantreiben, um unsere Arbeit, Freizeit und auch den Handel in die virtuelle Realität zu verlagern, führen zudem dazu, dass herstellende und Handelsunternehmen, denen keine virtuellen Produktreplikate vorliegen, von dieser Marktentwicklung des virtuellen Handels ausgeschlossen werden. Um dem entgegenzuwirken, wäre die Entwicklung einer Online-Plattform für virtuelle Produktreplikate mit eindeutigen Identifikationsmechanismen zielführend, um allen herstellenden und Handelsunternehmen den Zugang zum AR- und VR-basierten Handel mit Gütern zu ermöglichen. Es ist zu erwarten, dass Kooperationen zwischen beiden Industriezweigen mit der voranschreitenden Diffusion der AR- und VR-Technologien an Relevanz gewinnen werden. Neben semi-physischen und semi-virtuellen Gütern, bei denen das eine Gut jeweils als Träger des anderen fungiert, wird dabei die Bereitstellung von virtuellen Assets, welche die Wiederverwendung von 3DModellen in unterschiedlichen Prozessschritten ermöglichen und eine Schnittstelle für den, auch öffentlichen, Austausch und Handel von 3D-Modellen darstellen, eine maßgebliche Rolle spielen. 
3D-Modelle, die an den Schnittstellen beider Domänen zu finden sind, sind jedoch weitestgehend unerforscht. Es finden sich kaum Publikationen, welche die Wertigkeit, den Handel sowie den Erstellungsprozess und die unterschiedlichen Arten von virtuellen Assets oder semi-virtuellen und semi-physischen Gütern untersuchen, obwohl es im Falle von Ersterem Marktplätze gibt, auf denen diese angeboten und gehandelt werden (siehe Tab. 1). Das trifft ebenfalls auf Untersuchungen zur Übertragbarkeit von Methoden in die jeweils anderen Bereiche $\mathrm{zu}$, die nur vereinzelt zu finden sind. Auch die Mechanismen und Ansätze zur Einbindung des Nutzers als Schöpfer virtueller Güter stellen weiterhin eine interessante Forschungsrichtung dar, da der Grad der Einbindung das Geschäftsmodell von Spieleentwicklern dahingehend beeinflussen kann, dass der Nutzer als virtueller Entrepreneur die wertschöpfenden Prozesse innerhalb der Umgebungen übernimmt. Schlussendlich basiert das abgeleitete Modell zum Einsatz von 3D-Modellen in den Wertschöpfungsprozessen auf Angaben in der Literatur. Eine Validierung und Erweiterung des Modells basierend auf praktischer Empirie und Fallstudien mit Unternehmen aus beiden Bereichen ist daher obligatorisch. 


\section{Anhang}

\section{Herstellenden und Handelsunternehmen}

Wertschöpfungsphasen

\begin{tabular}{|c|c|c|c|}
\hline \multicolumn{2}{|l|}{ Entwicklung } & Produktion & \multirow[t]{2}{*}{ Vertrieb } \\
\hline \multicolumn{3}{|c|}{ Konzepte } & \\
\hline $\begin{array}{c}\text { Virtual } \\
\text { Prototyping }\end{array}$ & $\begin{array}{c}\text { Virtual } \\
\text { Collaboration }\end{array}$ & $\begin{array}{c}\text { Virtual } \\
\text { Fabrication }\end{array}$ & $\begin{array}{l}\text { Virtual Product } \\
\text { Experience }\end{array}$ \\
\hline $\begin{array}{l}\text { Artacho et al. 2010; Bao et al. } \\
\text { 2002; Bordegoni et al. 2006; } \\
\text { Hippel und Katz 2002; Israel et } \\
\text { al. 2009; Katicic et al. 2015; } \\
\text { Kim et al. 2011; Makris et al. } \\
\text { 2012; Park und Moon 2013; } \\
\text { Park et al. 2008; Stark et al. } \\
\text { 2010; Teklemariam und Das } \\
2017\end{array}$ & $\begin{array}{l}\text { Choi et al. 2002; } \\
\text { Heimrich und } \\
\text { Anderl 2016; } \\
\text { Pfouga und } \\
\text { Stjepandić 2015, } \\
\text { 2018; Rosenman } \\
\text { und Wang 2001; } \\
\text { Xiao et al. 2010; } \\
\text { Zhang et al. 2010 }\end{array}$ & $\begin{array}{l}\text { Bohm et al. 2005; } \\
\text { Dangelmaier et al. } \\
\text { 2005; Kang et al. } \\
2018\end{array}$ & $\begin{array}{l}\text { Algharabat et al. 2017; Bonetti et } \\
\text { al. 2018; Duffy et al. 2004; Füller } \\
\text { und Matzler 2007; Jiang und } \\
\text { Benbasat 2004; Klein 2003; Lu } \\
\text { und Smith 2010; Olsen und Saetre } \\
\text { 1997; Schnack et al. 2019; Tao et } \\
\text { al. 2018; Weisstein et al. 2016; Yi } \\
\text { et al. 2015; Yu und Damhorst } \\
\text { 2015; Yu et al. } 2012\end{array}$ \\
\hline \\
\hline \multicolumn{4}{|c|}{ Virtual Customer Integration } \\
\hline $\begin{array}{r}\text { tacho et al. 2010; Bugsha } \\
\text { Katicic et }\end{array}$ & 1 & ijller un & $\begin{array}{l}\text { 2007; Hippel und Katz 2002; } \\
\text { to et al. } 2018\end{array}$ \\
\hline
\end{tabular}

\section{Spieleentwickler}

Wertschöpfungsphasen

\begin{tabular}{|c|c|c|}
\hline Entwicklung & Produktion & Vertrieb \\
\hline & & Konzepte \\
\hline $\begin{array}{c}\text { Virtual } \\
\text { Prototyping }\end{array}$ & Virtual & Virtual Distribution \\
\hline Li und Kuo & Grimes 2015; & Animesh et al. 2011; Cheung et al. 2015; Cleghorn und Griffiths 2015; \\
$2007 ;$ Varajão & Ke et al. 2012; & Fairfield 2005; Gumussoy 2016; Guo und Barnes 2009, 2011; Hamari und \\
und Morgado & Varajão und & Keronen 2017; Hassouneh und Brengman 2011; Heimo et al. 2018; \\
2012 & Morgado 2012 & Jankowski et al. 2016; Lehdonvirta 2009; Lehdonvirta et al. 2009; \\
& & Mäntymäki und Salo 2015; Marder et al. 2019; Schmidt 2006; Shang et al. \\
& & 2012; Sheldon 2006; Strikwerda 2012; Wang und Chang 2014; Wu und Hsu \\
& & 2018; Zhu und Chang 2015 \\
\hline & \multicolumn{2}{|c|}{ Virtual Entrepreneurship and Customer Integration } \\
\hline
\end{tabular}

Abb. 3 Literaturkodierung 
Funding Open Access funding enabled and organized by Projekt DEAL.

Open Access Dieser Artikel wird unter der Creative Commons Namensnennung 4.0 International Lizenz veröffentlicht, welche die Nutzung, Vervielfältigung, Bearbeitung, Verbreitung und Wiedergabe in jeglichem Medium und Format erlaubt, sofern Sie den/die ursprünglichen Autor(en) und die Quelle ordnungsgemäß nennen, einen Link zur Creative Commons Lizenz beifügen und angeben, ob Änderungen vorgenommen wurden.

Die in diesem Artikel enthaltenen Bilder und sonstiges Drittmaterial unterliegen ebenfalls der genannten Creative Commons Lizenz, sofern sich aus der Abbildungslegende nichts anderes ergibt. Sofern das betreffende Material nicht unter der genannten Creative Commons Lizenz steht und die betreffende Handlung nicht nach gesetzlichen Vorschriften erlaubt ist, ist für die oben aufgeführten Weiterverwendungen des Materials die Einwilligung des jeweiligen Rechteinhabers einzuholen.

Weitere Details zur Lizenz entnehmen Sie bitte der Lizenzinformation auf http://creativecommons.org/ licenses/by/4.0/deed.de.

\section{Literatur}

Algharabat R, Alalwan AA, Rana NP, Dwivedi YK (2017) Three dimensional product presentation quality antecedents and their consequences for online retailers: the moderating role of virtual product experience. J Retail Consumer Serv 36:203-217

Animesh P, Yang O (2011) An Odyssey into virtual worlds: exploring the impacts of technological and spatial environments on intention to purchase virtual products. MISQ 35:789

Artacho MA, Ballester A, Alcántara E (2010) Analysis of the impact of slight changes in product formal attributes on user's emotions and configuration of an emotional space for successful design. J Eng Des 21:693-705

Bao JS, Jin Y, Gu MQ, Yan JQ, Ma DZ (2002) Immersive virtual product development. J Mater Process Technol 129:592-596

Bohm MR, Stone RB, Szykman S (2005) Enhancing virtual product representations for advanced design repository systems. J Comput Inf Sci Eng 5:360-372

Bonetti F, Warnaby G, Quinn L (2018) Augmented reality and virtual reality in physical and Online retailing: a review, synthesis and research agenda. In: Jung T, tom Dieck MC (Hrsg) Augmented reality and virtual reality. Springer, Cham, S 119-132

Bordegoni M, Colombo G, Formentini L (2006) Haptic technologies for the conceptual and validation phases of product design. Comput Graph 30:377-390

Bouchlaghem D, Shang H, Whyte J, Ganah A (2005) Visualisation in architecture, engineering and construction (AEC). Autom Constr 14:287-295

Bugshan H (2015) Co-innovation: the role of online communities. J Strateg Mark 23:175-186

Cheung CMK, Shen X-L, Lee ZWY, Chan TKH (2015) Promoting sales of online games through customer engagement. Electron Commer Res Appl 14:241-250

Choi ACK, Chan DSK, Yuen AMF (2002) Application of virtual assembly tools for improving product design. Int J Adv Manuf Technol 19:377-383

Cleghorn J, Griffiths M (2015) Why do gamers buy 'virtual assets'?: an insight in to the psychology behind purchase behaviour. Digit Educ Rev 27:91-110

Dahan E, Hauser JR (2002) The virtual customer. J Prod Innov Manag 19:332-353

Dangelmaier W, Fischer M, Gausemeier J, Grafe M, Matysczok C, Mueck B (2005) Virtual and augmented reality support for discrete manufacturing system simulation. Comput Ind 56:371-383

Duffy VG, Yen BPC, Cross GW (2004) Internet marketing and product visualization (IMPV) system: development and evaluation in support of product data management. Int J Comput Integr Manuf 17:1-15

Fairfield JAT (2005) Virtual property. Bust Univ Law Rev 85:1047-1102

Füller J, Matzler K (2007) Virtual product experience and customer participation-A chance for customercentred, really new products. Technovation 27:378-387

Grimes SM (2015) Playing by the market rules: promotional priorities and commercialization in children's virtual worlds. J Consumer Cult 15:110-134 
Gumussoy CA (2016) Acceptance of the virtual item auctioning system in online games; The role of intrinsic motivation, extrinsic motivation, and trust. Hum Factors Ergonomics Manuf Serv Ind 26:627-637

Guo Y, Barnes S (2009) Virtual item purchase behavior in virtual worlds: an exploratory investigation. Electron Commer Res 9:77-96

Guo Y, Barnes S (2011) Purchase behavior in virtual worlds: an empirical investigation in Second Life. Inf Manag 48:303-312

Hamari J, Keronen L (2017) Why do people buy virtual goods: a meta-analysis. Comput Human Behav 71:59-69

Hassouneh D, Brengman M (2011) Shopping in virtual worlds: perceptions, motivations, and behavior. J Electron Commer Res 12:320-335

Heimo OI, Harviainen JT, Kimppa KK, Mäkilä T (2018) Virtual to virtuous money: a virtue ethics perspective on video game business logic. J Bus Ethics 153:95-103

Heimrich F, Anderl R (2016) Approach for the visualization of geometric uncertainty of assemblies in CAD-systems. J Comput 11:247-257

von Hippel E, Katz R (2002) Shifting innovation to users via toolkits. Manage Sci 48:821-833

Israel JH, Wiese E, Mateescu M, Zöllner C, Stark R (2009) Investigating three-dimensional sketching for early conceptual design-Results from expert discussions and user studies. Comput Graph 33:462-473

Jankowski J, Bródka P, Hamari J (2016) A picture is worth a thousand words: an empirical study on the influence of content visibility on diffusion processes within a virtual world. Behav Inf Technol 35:926-945

Jiang Z, Benbasat I (2004) Virtual product experience: effects of visual and functional control of products on perceived diagnosticity and flow in electronic shopping. J Manag Inf Syst 21:111-147

Jung Y, Pawlowski SD (2014) Virtual goods, real goals: exploring means-end goal structures of consumers in social virtual worlds. Inf Manag 51:520-531

Jung Y, Pawlowski S (2015) The meaning of virtual entrepreneurship in social virtual worlds. Telematics Inform 32:193-203

Kang HS, Noh SD, Son JY, Kim H, Park JH, Lee JY (2018) The FaaS system using additive manufacturing for personalized production. Rapid Prototyp J 24:1486-1499

Katicic J, Häfner P, Ovtcharova J (2015) Methodology for emotional assessment of product design by customers in virtual reality. Presence: Teleoperators \& Virtual Environments 24:62-73

Ke D, Ba S, Stallaert J, Zhang Z (2012) An empirical analysis of virtual goods permission rights and pricing strategies. Decis Sci 43:1039-1061

Kim C, Lee C, Lehto MR, Yun MH (2011) Affective evaluation of user impressions using virtual product prototyping. Hum Factors Ergonom Manuf Serv Ind 21:1-13

Klein LR (2003) Creating virtual product experiences: the role of telepresence. J Interact Mark 17:41-55

Lee KH, Woo H, Suk T (2001) Data reduction methods for reverse engineering. Int J Adv Manuf Technol 17:735-743

Lehdonvirta V (2009) Virtual item sales as a revenue model: identifying attributes that drive purchase decisions. Electron Commer Res 9:97-113

Lehdonvirta V, Wilska T-A, Johnson M (2009) Virtual consumerism; case Habbo hotel. Inf Commun Soc $12: 1059-1079$

Li SG, Kuo X (2007) The enhanced quality function deployment for developing virtual items in massive multiplayer online role playing games. Comput Ind Eng 53:628-641

Lu Y, Smith S (2010) Augmented reality E-commerce system: a case study. J Comput Inf Sci Eng 10:21005

Makris S, Rentzos L, Pintzos G, Mavrikios D, Chryssolouris G (2012) Semantic-based taxonomy for immersive product design using VR techniques. CIRP Ann 61:147-150

Mäntymäki M, Salo J (2015) Why do teens spend real money in virtual worlds? A consumption values and developmental psychology perspective on virtual consumption. Int J Inf Manage 35:124-134

Marder B, Gattig D, Collins E, Pitt L, Kietzmann J, Erz A (2019) The Avatar's new clothes: Understanding why players purchase non-functional items in free-to-play games. Comput Human Behav 91:72-83

Olsen KA, Saetre P (1997) Managing product variability by virtual products. Int J Prod Res 35:2093-2108

Park H, Moon H-C (2013) Design evaluation of information appliances using augmented reality-based tangible interaction. Comput Ind 64:854-868

Park H, Son J-S, Lee K-H (2008) Design evaluation of digital consumer products using virtual realitybased functional behaviour simulation. J Eng Des 19:359-375

Pfouga A, Stjepandić J (2015) Leveraging 3D CAD data in product life cycle: exchange_-visualizationcollaboration. In: Curran R, Wognum N, Borsato M, Stjepandić J, Verhagen WJC (Hrsg) Transdisciplinary lifecycle analysis of systems. IOS Press, Amsterdam, S 575-584 
Pfouga A, Stjepandić J (2018) Leveraging 3D geometric knowledge in the product lifecycle based on industrial standards. J Comput Des Eng 5:54-67

Rengier F, Mehndiratta A, von Tengg-Kobligk H, Zechmann CM, Unterhinninghofen R, Kauczor H-U, Giesel FL (2010) 3D printing based on imaging data: review of medical applications. Int J CARS 5:335-341

Rosenman M, Wang F (2001) A component agent based open CAD system for collaborative design. Autom Constr 10:383-397

Schmidt AU (2006) Multi-level markets and incentives for information goods. Inf Econ Policy 18:125-138

Schnack A, Wright MJ, Holdershaw JL (2019) Immersive virtual reality technology in a three-dimensional virtual simulated store: investigating telepresence and usability. Food Res Int 117:40-49

Scopigno R, Callieri M, Cignoni P, Corsini M, Dellepiane M, Ponchio F, Ranzuglia G (2011) 3D models for cultural heritage: beyond plain visualization. Computer 44:48-55

Shang R-A, Chen Y-C, Huang S-C (2012) A private versus a public space: anonymity and buying decorative symbolic goods for avatars in a virtual world. Comput Human Behav 28:2227-2235

Sheldon DP (2006) Claiming ownership, but getting owned: contractual limitations on asserting property interests in virtual goods. UCLA Law Rev 54:751-787

Stark R, Israel JH, Wöhler T (2010) Towards hybrid modelling environments-merging desktop-CAD and virtual reality-technologies. CIRP Ann 59:179-182

Strikwerda L (2012) Theft of virtual items in online multiplayer computer games: an ontological and moral analysis. Ethics Inf Technol 14:89-97

Tao X, Chen X, Zeng X, Koehl L (2018) A customized garment collaborative design process by using virtual reality and sensory evaluation on garment fit. Comput Ind Eng 115:683-695

Teklemariam HG, Das AK (2017) A case study of phantom omni force feedback device for virtual product design. Int J Interact Des Manuf 11:881-892

Varajão J, Morgado L (2012) Potential of virtual worlds for marketing tests of product prototypes. J Text Inst 103:960-967

Wang W-T, Chang W-H (2014) A study of virtual product consumption from the expectancy disconfirmation and symbolic consumption perspectives. Inf Syst Front 16:887-908

Webster J, Watson RT (2002) Analyzing the past to prepare for the future: writing a literature review. MIS Q 26:xiii-xxiii

Weisstein FL, Kukar-Kinney M, Monroe KB (2016) Determinants of consumers' response to pay-whatyou-want pricing strategy on the Internet. J Bus Res 69:4313-4320

Wu S-L, Hsu C-P (2018) Role of authenticity in massively multiplayer online role playing games (MMORPGs): determinants of virtual item purchase intention. J Bus Res 92:242-249

Xiao S, Xudong C, Li Z, Guanghong G (2010) Modeling framework for product lifecycle information. Simul Model Pract Theory 18:1080-1091

Yi C, Jiang Z, Benbasat I (2015) Enticing and engaging consumers via online product presentations: the effects of restricted interaction design. J Manag Inf Syst 31:213-242

Yu U-J, Damhorst ML (2015) Body satisfaction as antecedent to virtual product experience in an online apparel shopping context. Cloth Text Res J 33:3-18

Yu U-J, Lee H-H, Damhorst ML (2012) Exploring multidimensions of product performance risk in the online apparel shopping context. Cloth Text Res J 30:251-266

Zhang H, Wang H, Chen D, Zacharewicz G (2010) A model-driven approach to multidisciplinary collaborative simulation for virtual product development. Adv Eng Informatics 24:167-179

Zhu DH, Chang YP (2015) Effects of interactions and product information on initial purchase intention in product placement in social games: the moderating role of product familiarity. J Electron Commer Res 16:22-33 